\title{
Segments of spiral arms of the Galaxy traced by classical Cepheids: Effects of age heterogeneity
}

\author{
Angelina V. Veselova, Igor' I. Nikiforov \\ Department of Celestial Mechanics, Saint Petersburg State University, Universitetskij \\ Prospekt 28, Staryj Peterhof, Saint Petersburg, 198504Russia; linav93@yandex.ru (AVV)
}

\begin{abstract}
We investigated the dependence of the parameters of the segments of spiral arms of the Galaxy on the age of classical Cepheids. The catalog of Cepheids (Mel'nik et al. 2015) was divided into two samples - relatively young $\left(P>9^{\mathrm{d}}\right)$ and relatively old $\left(P<9^{\mathrm{d}}\right)$ objects. The parameters of the spiral structure were determined both for two samples separately and jointly for the combination of two systems of segments traced by young and old objects. For most of the segments, their parameters for young and old objects differ significantly. Taking into account the difference between the two segment systems, we obtained the estimate $R_{0}$ equal to $7.23_{-0.18}^{+0.19} \mathrm{kpc}$, which in the modern LMC calibration corresponds to the value of $R_{0}=\left.\left.8.08_{-0.20}^{+0.21}\right|_{\text {stat. }}{ }_{-0.36}^{+0.38}\right|_{\text {cal. }}$ kpc. It is shown that the displacement between the segments is not reduced to the effect of differential rotation only. To interpret this displacement for objects of Perseus and Sagittarius-2 segments we carried out a dynamic modeling of the change in the position of the segment points when moving in the smooth gravitational field of the Galaxy. At the angular velocity of rotation of the spiral pattern $\Omega_{\mathrm{p}}=25.2 \pm 0.5 \mathrm{~km} \mathrm{~s}^{-1} \mathrm{kpc}^{-1}$ (Dambis et al. 2015) the observed displacement between segments on young and old objects can be explained by the amplitude of spiral perturbations of the radial velocity of $u=10 \pm 1.5 \mathrm{~km} \mathrm{~s}^{-1}$. For the constructed double system of spiral segments, it is demonstrated that the assumption of constancy of the pitch angles within each segment and the assumption that the pole of the spiral pattern is in the direction of the nominal center of the Galaxy do not contradict the data within the range of uncertainty.
\end{abstract}

Keywords: Galaxy: fundamental parameters - Galaxy: structure. 


\section{Introduction}

The spiral structure is a significant feature of many disk galaxies. The spiral arms, that is, the branches of the spiral pattern, are visible against the background of the galactic disk as narrow elongated areas of increased brightness, delineated by regions of intense star formation. The presence of spiral arms in the Milky Way has been known since the mid-20th century (e.g., van de Hulst et al. 1954), but the question of the morphology of the spiral structure is still not fully clarified (see, e.g., a brief review in Nikiforov \& Veselova 2018a).

The most common model of a spiral arm is the logarithmic spiral, with a single value of the pitch angle $i$ for all the arms or with a different value for each arm. However, the number of arms in different models of the Galaxy may be different, and the specified number of arms largely determines the value of $i$. For example, Francis \& Anderson (2012) adopted a two-arm logarithmic model of a spiral structure with a constant pitch angle $i=-5.56 \pm 0$.06, which was the same for both arms traced by giant molecular clouds, H II regions, 2MASS sources and H I distribution for Galactocentric distances up to 12-15 kpc. On the other hand, Vallée (2008), based on the analysis and comparison of a number of works, suggested a four-arm logarithmic model with a pitch angle $i=-12$ :8 as the most suitable model of a spiral pattern, consistent with the tangential directions to the arms determined by observations of $\mathrm{H} \mathrm{II},{ }^{12} \mathrm{CO},{ }^{13} \mathrm{CO}$, and ${ }^{26} \mathrm{Al}$. According to a survey in Vallée (2015), published pitch angle values, depending on the accepted number of arms, cover from $-6^{\circ}$ to $-28^{\circ}$ for the global Galaxy.

In many studies, the spiral pattern is assumed to be symmetrical: the arms pass into each other when turning around the Galactic center at an angle of $180^{\circ}$ in the case of a two-arm pattern or $90^{\circ}$ in the case of a four-arm pattern. This assumption, however, leads to an additional condition: the pitch angles of the arms in this case must be equal. Efremov (2011) based on the analysis of data on the distribution of neutral, molecular and ionized hydrogen suggested the presence of a symmetrical logarithmic four-arm spiral pattern in the inner part of the Galaxy, but noted that the symmetry may be broken in the outer regions.

In recent years, the assumption of equal pitch angles for all arms has been gradually abandoned. For example, in Bobylev \& Bajkova (2014) the value of the pitch angle for four segments of the arms is estimated based on data on the spatial distribution of masers and very young open clusters in the Outer arm. The resulting pitch angles of individual segments are compatible with each other and close to $i=-13^{\circ} \pm 1^{\circ}$.

However, the results of a number of studies suggest the possibility of a significantly more complex morphology. Lépine et al. (2001) considered the spiral pattern as a superposition of two- and four-arm models with different pitch angles ( $6^{\circ}$ for two-arm and $12^{\circ}$ for four-arm); thus, the total number of spiral arms is six. The proposed model better satisfies the data on Cepheid kinematics than the two-arm and four-arm models separately and can explain the possible bifurcation of arms, an example of which can be observed in the galaxy M 101. Englmaier et al. (2011) according to the data on the distribution of 
neutral hydrogen suggest the following model of a spiral pattern: in the inner part of the Galaxy, two significant arms start from the ends of the bar, then, at a Galactocentric distance of about $7.8 \mathrm{kpc}$, the two-arm pattern splits into four arms, continuing to a distance of about $20 \mathrm{kpc}$. The bifurcation points were not $180^{\circ}$ apart; one of the branching points is presumably located near the Sun, which may affect the velocity distribution in the solar neighborhood.

The problem of determining the parameters of the Galactic spiral structure can also be associated with the problem of determining the distance to the Galactic center $\left(R_{0}\right)$ under the assumption that the Galactic center is the pole of the spiral arms. Fixing the value of $R_{0}$ is a standard assumption in studies of the spiral structure (e.g., in the mentioned works $R_{0}=7.5-8.0 \mathrm{kpc}$ ). Due to complex correlations with other parameters of spiral arms (see Nikiforov \& Veselova 2018a), this creates an additional source of systematic errors, different for different subsystems due to mismatch of methods for determination of heliocentric distances and mismatches (at least for calibrations) of photometric distance scales. Freeing $R_{0}$ when modeling the spiral structure largely removes these problems. On the other hand, this gives another independent method for determining $R_{0}$. The question of a reliable value of $R_{0}$ cannot be considered closed with confidence, since modern estimates still differ significantly from each other. For example, Braga et al. (2018) based on data on Cepheids in the bulge obtained an estimate of $R_{0}=8.46 \pm 0.03$ (stat.) \pm 0.11 (sys.) kpc; according to data on S0-2 star orbiting the supermassive black hole at the Galactic center GRAVITY Collaboration et al. (2019) presented an estimate of $R_{0}=8.178 \pm 0.013$ (stat.) \pm 0.022 (sys.) kpc, and Do et al. (2019) gave a value of $7.946 \pm 0.050$ (stat) \pm 0.032 (sys) kpc.

In our previous works (Nikiforov \& Veselova 2015, 2018a) we proposed a new approach to determining the geometric parameters of the Milky Way spiral arm segments from the spatial distribution of tracing objects: in an effort to minimize assumptions, we do not assume or determine the number of spiral arms, but rather estimate the parameters of individual detected segments of arms, considering the geometric pole to be the same for all segments and obtaining the parameter $R_{0}$ together with the geometric parameters, such as the pitch angles and the positional parameters of the segments. The method was tested on maser source data, and we obtained a solar Galactocentric distance estimate of $R_{0}=8.8 \pm 0.5 \mathrm{kpc}$. Our numerical experiments (Nikiforov \& Veselova 2018b) have confirmed the effectiveness of the algorithm developed by us for a wide range of possible parameter values and made it meaningful to develop a more complex method that will take into account the influence of distance uncertainties and the natural scatter of objects across the segments.

Using the maximum likelihood method we developed an algorithm for the spatial modeling of spiral arm segments taking into account the natural dispersion across the segment and the uncertainty of the distance modulus, $d$. The algorithm does not require the initial strict assignment of the object to a specific segment. In Veselova \& Nikiforov (2018) the proposed method was applied to data on the spatial distribution of classical Cepheids from the catalog in Mel'nik et al. (2015). The initial assignment of objects to 
segments was carried out by analyzing the distribution of $X_{\mathrm{s}}$ coordinates, where $X_{\mathrm{s}}$ is the abscissa of the intersection point of the logarithmic spiral corresponding to the object's phase by the direction to the Galactic center.

In this paper, based on the developed algorithm (Sect. 21), we find out whether the age heterogeneity of classical Cepheids is a significant factor in the spatial modeling of spiral segments traced by these objects, and then investigate the detected age effects. In Sect. 3, we determine the parameters of the spiral structure for old and young Cepheids separately and together, allowing for a distinction between two systems of segments traced by young and old objects. Then we perform a dynamic modeling to interpret the detected displacement between the Perseus and Sagittarius-2 segments in the two systems (Sect. 4). In Sect. 5, we show how a fixed value of $R_{0}$ affects the estimates of the pitch angles, and test the possibility of rejecting other standard assumptions when studying the spiral structure - the constancy of the pitch angles and the coincidence of the arms pole with the Galactic center.

\section{The method of modeling the spiral arm segments}

\subsection{Likelihood function for a set of segments}

We investigate the distribution of objects in the projection on the Galactic plane in the Cartesian coordinate system. The model of the center line of the segment depends on a number of parameters $(\vec{p})$ and represents the dependence of the Galactoaxial distance on the Galactocentric longitude $\lambda$ measured from the sunward direction clockwise:

$$
R_{\mathrm{mod}}=R_{\mathrm{mod}}(\lambda, \vec{p})
$$

We assume that the displacement $w$ of the object across the segment (for which the shape of the center line is given by the model) and the error of the distance modulus obey the normal distribution, so the likelihood function is the product of the corresponding distribution functions, taking into account the uniform dispersion $\sigma_{\mathrm{w}}$ across the arm and the uncertainty of the distance modulus $\sigma_{d}$. For one segment, the likelihood function is given as

$$
L=\prod_{j=1}^{N} \frac{1}{\sqrt{2 \pi} \sigma_{w}} \exp \left[-\frac{w^{2}\left(d_{0, j}, \vec{p}\right)}{2 \sigma_{w}^{2}}\right] \frac{1}{\sqrt{2 \pi} \sigma_{d}} \exp \left[-\frac{\left(d_{\mathrm{obs}, j}-d_{0, j}\right)^{2}}{2 \sigma_{d}^{2}}\right]
$$

but for convenience we use the negative log likelihood function $\mathfrak{L}$ represented as

$$
\mathfrak{L}=-\ln L=N \ln (2 \pi)+N \ln \sigma_{d}+N \ln \sigma_{w}+\frac{1}{2} \sum_{j=1}^{N} \underbrace{\min _{d_{0, j}}\left[\frac{w^{2}\left(d_{0, j}, \vec{p}\right)}{\sigma_{w}^{2}}+\frac{\left(d_{\mathrm{obs}, j}-d_{0, j}\right)^{2}}{\sigma_{d}^{2}}\right]}_{S_{j}^{2}},
$$

where $N$ is the number of objects, $d_{\mathrm{obs}, j}$ represents the observed (measured) distance modulus of the $j$-th object and $d_{0, j}$ is its reduced distance modulus (see Fig. 1). The 


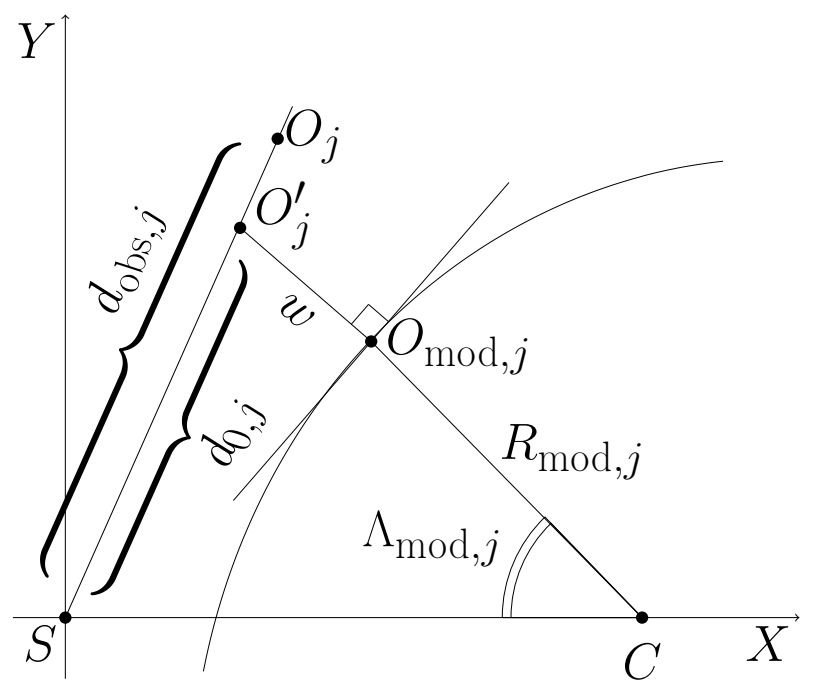

Fig. 1. Location of an object relative to the center line of a spiral segment (curved line). The Sun is placed at the origin of the Cartesian coordinate system, $X Y$ is the Galactic plane and $C$ represents the Galactic center. $O_{j}$ is the observed position of the $j$-th object, $O_{j}^{\prime}$ signifies the reduced position of the same object, $O_{\bmod , j}$ is the point on a center line for which the distance $w$ from reduced position is minimal (the "model position" of the object) and $\Lambda_{\text {mod }, j}$ is the Galactocentric longitude of the point $O_{\bmod , j}$.

expression in square brackets can be considered as the square of weighted distance from the reduced position of the $j$-th object to the center line of the segment at the accepted value of $d_{0, j}$. The calculation of the segment parameters is performed as follows: first, we find the minimum value $S_{j}^{2}$ of the square of weighted distance by varying the reduced distance modulus $d_{0, j}$ for each $j$-th object at a given set of parameters $\vec{p}$, i.e. we determine the reduced position $O_{j}^{\prime}$ of an observed position $O_{j}$ of the $j$-th object by shifting it on the line of sight so that for $O_{j}^{\prime}$ the weighted distance is minimal, $O_{\bmod , j}$ is the point on a center line for which the distance $w$ from reduced position is minimal, in fact, this is a projection of the reduced position $O_{j}^{\prime}$ on the model spiral. Next we can determine the overall minimum $\mathfrak{L}_{0}$ for the segment while minimizing its parameters $\vec{p}$. The shape of the center line of the segment determines the value of the weighted distance.

When considering a set of segments, the likelihood function takes the form of a product of functions for individual segments. We assume $\sigma_{d}$ to be the same for the entire sample of objects, but $\sigma_{w}$ has its own value $\left(\sigma_{w, a}\right)$ for each segment. For a set of $N_{\text {segm }}$ segments the negative log likelihood function is represented as

$\mathfrak{L}=N \ln (2 \pi)+N \ln \sigma_{d}+\sum_{a=1}^{N_{\text {segm }}} N_{a} \ln \sigma_{w, a}+\frac{1}{2} \sum_{a=1}^{N_{\text {segm }}} \sum_{j=1}^{N_{a}} \min _{d_{0, j}}\left[\frac{w^{2}\left(d_{0, j}, \overrightarrow{p_{a}}\right)}{\sigma_{w, a}^{2}}+\frac{\left(d_{\mathrm{obs}, j}-d_{0, j}\right)^{2}}{\sigma_{d}^{2}}\right]$.

Here $N$ is the total number of objects, $N_{a}$ is the number of objects tracing the $a$-th segment and $\overrightarrow{p_{a}}$ is the set of parameters of the $a$-th segment 


\subsection{Likelihood function in case of a logarithmic model}

The assumption about the model of the segment center line is applied when we calculate the weighted distances. We have chosen the logarithmic spiral as the most popular model. We assume the spiral to be in the Galactic plane and the direction from the Sun to the spiral pole (to the Galactic center) to be known. The spiral arm is then represented by a segment of the logarithmic spiral

$$
R_{\text {mod }}\left(\lambda ; R_{0}, k, X_{\mathrm{s}}\right)=\left|R_{0}-X_{\mathrm{s}}\right| \mathrm{e}^{k \lambda} .
$$

Here, $\lambda \in(-\infty,+\infty)$ is the Galactocentric longitude (it is measured from the sunward direction clockwise when viewed from the North Galactic Pole); $k \equiv \tan i$, where $i$ is the pitch angle (it is negative for a trailing segment); $X_{\mathrm{s}}$ is the abscissa of the point, at which the segment intersects the direction to the Galactic center. In the sunward direction $\lambda=0 \pm 2 \pi n, n \in \mathbb{Z}$.

In general, we assume a value of the solar Galactocentric distance $R_{0}$ to be common for all segments, but the pitch angles, $X_{\mathrm{s}}$ values and dispersions $\sigma_{w}$ may differ for different segments. For a logarithmic model the likelihood function $\mathfrak{L}$ for a set of segments takes the form

$$
\begin{aligned}
\mathfrak{L}=N \ln (2 \pi)+N \ln \sigma_{d} & +\sum_{a=1}^{N_{\mathrm{segm}}} N_{a} \ln \sigma_{w, a}+ \\
& +\frac{1}{2} \sum_{a=1}^{N_{\mathrm{segm}}} \sum_{j=1}^{N_{a}} \min _{d_{0, j}}\left[\frac{w^{2}\left(d_{0, j} ; R_{0}, i_{a}, X_{\mathrm{s}, a}\right)}{\sigma_{w, a}^{2}}+\frac{\left(d_{\mathrm{obs}, j}-d_{0, j}\right)^{2}}{\sigma_{d}^{2}}\right] .
\end{aligned}
$$

To determine the distance $w$ from the reduced position $O_{j}^{\prime}$ of the $j$-th object with the coordinates $\left(X_{0, j}, Y_{0, j}\right)$ to the spiral, the roots of the following transcendental equation on the longitude $\Lambda_{\text {mod, } j}$ of the point on the spiral with the minimum distance to $O_{j}^{\prime}$ are calculated:

$$
\begin{aligned}
\left(X_{0, j}-R_{0}\right)\left(\sin \Lambda_{\text {mod }, j}-k_{a} \cos \Lambda_{\text {mod }, j}\right)-k_{a} \mid & R_{0}-X_{\mathrm{s}, a} \mid \mathrm{e}^{k_{a} \Lambda_{\bmod , j}}+ \\
& +Y_{0, j}\left(k_{a} \sin \Lambda_{\text {mod }, j}+\cos \Lambda_{\text {mod }, j}\right)=0,
\end{aligned}
$$

where $k_{a} \equiv \tan i_{0, a}$. Optimal parameters of a set of segments are determined by minimizing the function $\mathfrak{L}$. In this way, we jointly obtain the values of $R_{0}$, pitch angles $i_{a}$, parameters $X_{\mathrm{s}, a}$ and dispersions $\sigma_{w, a}$ for $a=1,2, \ldots, N_{\mathrm{segm}}$. Generally the number of optimized values is $M=3 N_{\text {segm }}+1$.

In the case of far or sparsely populated segments the deviation of objects from the center line of the segment can be completely explained by only the presence of uncertainty in the distance moduli. For these segments we set $\sigma_{w}=0$ and the negative likelihood function takes the form

$$
\mathfrak{L}=\frac{N}{2} \ln (2 \pi)+N \ln \sigma_{d}+\frac{1}{2} \sum_{j=1}^{N} \underbrace{\min _{d_{0, j}} \frac{\left(d_{\mathrm{obs}, j}-d_{0, j}\right)^{2}}{\sigma_{d}^{2}}}_{S_{j}^{2}} .
$$




\subsection{Estimation of confidence intervals}

The boundaries of the confidence interval of the parameter $p_{j}$ for the confidence level $1 \sigma$ can be determined from the equation

$$
\mathfrak{L}_{\mathrm{m}}\left(p_{j}\right)=\mathfrak{L}_{0}+\frac{1}{2}, \quad \mathfrak{L}_{0}=\min \mathfrak{L}, \quad \mathfrak{L}_{\mathrm{m}}\left(p_{j}\right)=\min _{p_{j}=\text { const }} \mathfrak{L} .
$$

Here $\mathfrak{L}_{0}$ is the minimum of the likelihood function, and $\mathfrak{L}_{\mathrm{m}}$ is the profile of the log likelihood function for the parameter $p_{j}$ obtained by optimizing all parameters except $p_{j}$.

To reduce the computation time in our work, the boundaries of confidence intervals were determined in the parabolic approximation. We consider the deviation $\Delta p_{j}>0$ of the parameter $p_{j}$ from the optimal value $p_{j}^{0}$, then determine the values of $\mathfrak{L}_{\mathrm{m}}$ at fixed $p_{j}^{-}=p_{j}^{0}-\Delta p_{j}$ and $p_{j}^{+}=p_{j}^{0}+\Delta p_{j}$, and then find the differences $\sigma^{+}$and $\sigma^{-}$between the optimal value $p_{j}^{0}$ and the boundaries of the confidence interval

$$
\sigma^{-}=\frac{\Delta^{2} p_{j}}{2\left(\mathfrak{L}_{\mathrm{m}}\left(p_{j}^{-}\right)-\mathfrak{L}_{0}\right)}, \quad \sigma^{+}=\frac{\Delta^{2} p_{j}}{2\left(\mathfrak{L}_{\mathrm{m}}\left(p_{j}^{+}\right)-\mathfrak{L}_{0}\right)} .
$$

\subsection{Assignment of objects to spiral segments}

There may be a question about the correct assignment of objects to spiral segments. We have proposed and implemented (Veselova \& Nikiforov 2018) the following algorithm. The initial division of objects into segments is carried out in accordance with the minima of the distribution function of the values of $X_{\mathrm{s}}$ which are computed for every object under the assumption of $R_{0}$ and $i_{0}$ values on a grid. Next we identify the basic sample of objects whose assignment to segments does not depend on assumptions on $R_{0}$. Then we optimize the parameters of segments traced by the basic sample by minimizing $\mathfrak{L}$, and other objects are assigned to the segments in accordance with the minimum weighted distance: for each object we compute the $S_{j}^{2}$ values relative to each segment and assign the object to the segment with minimal $S_{j}^{2}$. Next, the iterative reassignment of objects to specific segments and the optimization of parameters for the final assignment of objects into segments are carried out.

We chose classical Cepheids as objects that trace the spiral structure. In Veselova \& Nikiforov (2018) and in current work we utilized data from the catalog in Mel'nik et al. (2015) (see Sect. 3 for details). Analysis of the spatial distribution of 565 Cepheids from this catalog allowed Dambis et al. (2015) to identify four segments of the global spiral structure. In Veselova \& Nikiforov (2018) we aimed to undertake a more detailed analysis of the nearest spiral structure and we also considered slightly larger region populated by Cepheids. According to the algorithm described above, we investigated the location of minima of the distribution function of $X_{\mathrm{s}}$ values for different values of $R_{0}$ and for models with different numbers of segments. Figure 2(a) and 2(b) depicts the $X_{\mathrm{s}}$ distribution for models with seven and eight segments. The analysis of the consistency of the model and 

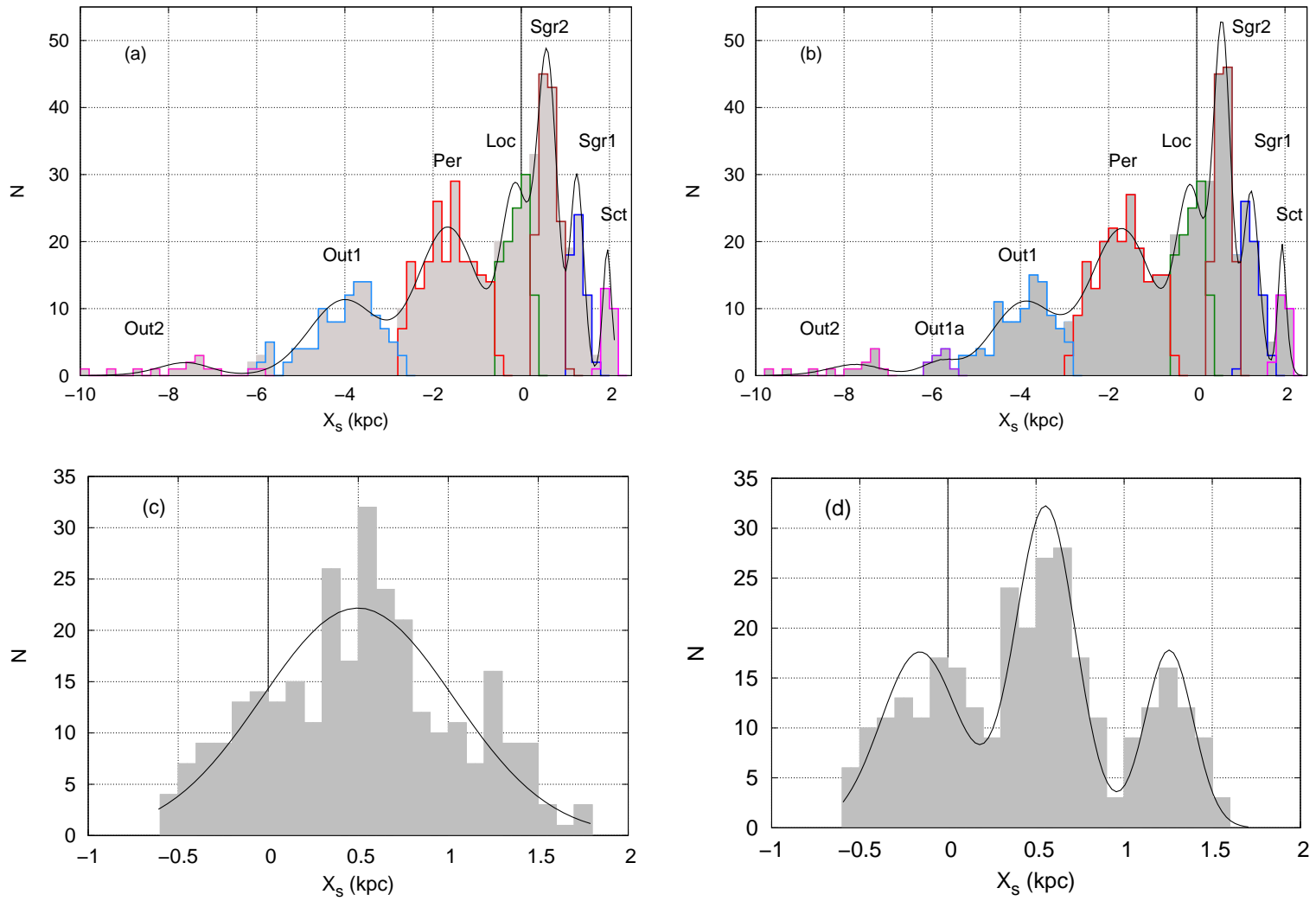

Fig. 2. Distribution of $X_{\mathrm{S}}$ values in the cases of seven (a) and eight (b) segments in the model, taken from Veselova \& Nikiforov (2018); the $X_{\mathrm{S}}$ distributions in the case of a single Sagittarius arm (c) and when dividing it into three separate segments (d), constructed only from the Cepheids tracing these details. The solid line displays the model function of the distribution of values $X_{\mathrm{s}}$ calculated with the uncertainty in distance moduli taken into account. In each panel, the position of the Sun corresponds to $X_{\mathrm{s}}=0.0 \mathrm{kpc}$ as marked by the solid vertical line. 
observed distribution functions, conducted applying the Pearson $\left(\chi^{2}\right)$ criterion, indicated the preference for a model with eight segments.

Thus, according to the data on 636 Cepheids, we preferred an eight segment model of nearby spiral structure. In comparison with the set of segments presented in Dambis et al. (2015), we divided the single Sagittarius arm into three segments (Local arm, Sagittarius-1 and Sagittarius-2) and, considering a slightly larger spatial region populated by Cepheids, we also added a more distant arm (Outer-2) and identified a small segment (Outer-1a). In Figure 2(c) and 2(d), we compare the model with a single Sagittarius arm and the model with three distinct segments. According to the Pearson criterion, the model with a single segment is rejected. Note that we did not draw conclusions about the global spiral structure, and only discussed the parameters of individual segments.

\section{Determination of parameters of segments traced by classical Cepheids}

In this paper we compare the parameters of spiral segments traced by young and old classical Cepheids from the catalog in Mel'nik et al. (2015). This catalog contains data on coordinates, proper motions, radial velocities, pulsation periods, and apparent stellar

magnitudes of 674 Cepheids. Authors did not specify the possible value of the distance modulus error. In our work we assumed $\sigma_{d}=0.14^{m}$, based on comparisons with other distance catalogs, as well as a sharp restriction from below on $\sigma_{d}$ created by a very narrow Scutum arm. This value allowed us to determine the value of dispersions across the arm in fairly populated segments. We discuss the possible influence of adopted $\sigma_{d}$ on parameters of segments later in this section.

According to the distance scale utilized in the catalog, the distance modulus of the Large Magellanic Cloud (LMC) is $d_{\mathrm{LMC}}=18.25 \pm 0.05$ (Berdnikov et al. 2000). We rescale the final $R_{0}$ estimate obtained by us to the modern LMC calibration $d_{\mathrm{LMC}}=18.49 \pm 0.09$ (de Grijs et al. 2014), i.e. we use a correction factor of 1.117 (Sect. 6). Other parameters of spiral segments obtained in this work should also be multiplied by this factor.

As had been demonstrated by Karimova \& Pavlovskaya (1974), the distribution of classical Cepheids over pulsation periods has a minimum near the period of $9^{\mathrm{d}}$ (see also Fig. 3a, based on the data we used). Cepheids with smaller values of the period have a greater age, then we call the selection of such objects old, and the rest of the objects are called young. In total, the sample considered consisted of 140 young and 494 old objects.

According to different relations for the age dependence on the pulsation period, we get slightly different age estimates for the period of $9 \mathrm{~d}$. According to the relation in Efremov (2003), the age estimate is $\sim 7.6 \times 10^{7} \mathrm{yr}$. If we apply the relation in Joshi \& Joshi (2014), we obtain an estimate of $\sim 7.3 \times 10^{7} \mathrm{yr}$. The median pulsation period for the old sample is nearly $4.6 \mathrm{~d}$, and if we consider several modern relations, we conclude that this value corresponds to the age of $1 \times 10^{8} \mathrm{yr}$, and the median period for the young sample $-13.5 \mathrm{~d}$ - corresponds to the age of $5 \times 10^{7} \mathrm{yr}$.

The spatial distribution of young and old Cepheids is presented in Fig. 3(b). We can see that young objects are situated mostly inside the solar circle, but outside the solar 

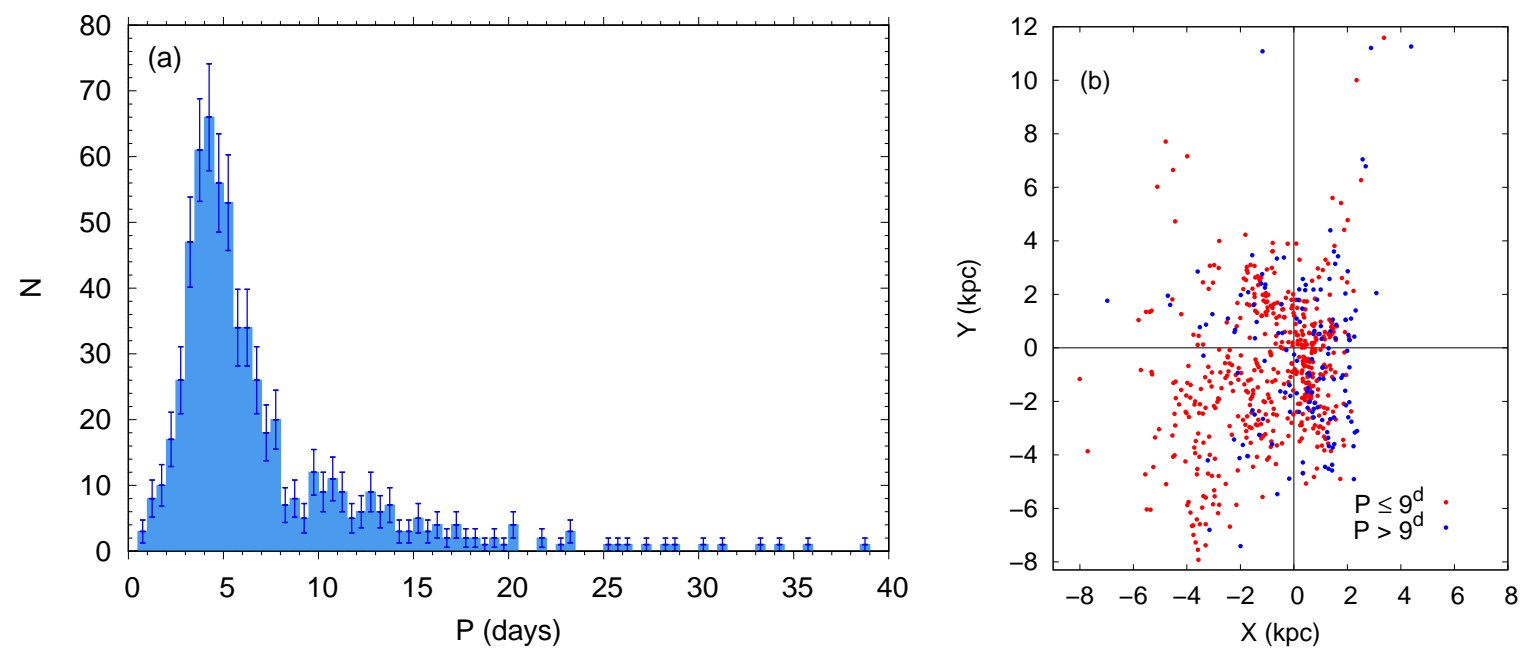

Fig. 3. Distribution of pulsation periods (a) and spatial distribution of old and young Cepheids projected on the Galactic plane (b). Old Cepheids are displayed in red and young Cepheids are marked in blue. The Sun is placed at $X=0.0 \mathrm{kpc}, Y=0.0 \mathrm{kpc}$.

circle they are located very sparsely. The nearest to the Galactic center segment (Scutum arm) consists mostly of young Cepheids, but the Outer segments are mostly represented by old objects.

In accordance with the previously mentioned algorithm, the samples were divided into segments. It was revealed that young objects trace only seven separate segments (there is no segment Outer-1a), and old objects form eight segments. Note that when optimizing the parameters separately for each of two samples, the estimates of $R_{0}$ are similar: $R_{0}=7.15 \pm 0.24 \mathrm{kpc}$ for young objects and $R_{0}=7.33 \pm 0.29 \mathrm{kpc}$ for old objects. The similarity of these values provides a basis for joint optimization of the parameters of two spiral patterns traced by young and old Cepheids. The results of the simultaneous optimization of the parameters for two samples are presented in Table 1, and the value $R_{0}$ is equal to $7.23_{-0.18}^{+0.19} \mathrm{kpc}$. Table 2 lists the differences between the parameters of segments traced by old and young Cepheids. Difference $\Delta i_{\mathrm{o}-\mathrm{y}}$ is significant for the Sagittarius-1 and Sagittarius- 2 arms, for the Local arm significance is marginal because $\Delta i_{\text {o-y }}$ is only greater than $2 \sigma$. Difference $\Delta X_{\mathrm{s}, \mathrm{o}-\mathrm{y}}$ in the $X_{\mathrm{s}}$ values is significant for the Sagittarius-2 arm, but for the Local and Perseus arms significance is marginal.

In Figure 4 we compare the $1 \sigma$ confidence regions for old and young segments. We consider only those segments that are quite large and have significant differences in parameters of center lines. One can see that for all three cases confidence regions do not intersect near the $X$-axis, so we can say that the young and old segments differ significantly.

The center lines of all traced segments in the projection on the $X Y$ plane are presented in Figure 5. Dashed lines correspond to segments traced by young objects, solid lines - for the old ones. One can see that in some cases the center lines of the young and old segments intersect in the region inhabited by Cepheids.

Figure 6] shows the dependence of the $R_{0}$ estimate on the assumed value of uncertainty 
Table 1. Parameters of spiral segments traced by young and old Cepheids. A zero width value $\sigma_{w}$ indicates that the deviation of the object from the center line of the segment can be explained by the uncertainty in distance moduli

\begin{tabular}{|c|c|c|c|c|c|c|}
\hline Segment & $i$ & $\begin{array}{c}\text { Young } \\
X_{\mathrm{s}}(\mathrm{kpc})\end{array}$ & $\sigma_{w}(\mathrm{kpc})$ & $i$ & $\begin{array}{c}\text { Old } \\
X_{\mathrm{s}}(\mathrm{kpc})\end{array}$ & $\sigma_{w}(\mathrm{kpc})$ \\
\hline Scutum & $-11.7_{-0.9}^{+0.9}$ & $2.01_{-0.03}^{+0.04}$ & $(0.00)$ & $-8.9_{-2.1}^{\circ} .2^{\circ}$ & $1.90_{-0.06}^{+0.06}$ & $(0.00)$ \\
\hline Sagittarius-1 & $-13.1_{-1.4}^{+1.4}$ & $1.30_{-0.05}^{+0.05}$ & $0.214_{-0.032}^{+0.033}$ & $-8.3_{-0.8}^{+0.8}$ & $1.25_{-0.02}^{+0.02}$ & $0.119_{-0.017}^{+0.017}$ \\
\hline Sagittarius-2 & $-10.9^{+0.6}$ & $0.699_{-0.022}^{+0.023}$ & $0.065_{-0.013}^{+0.013}$ & $-7.4_{-0.7}^{+0.7}$ & $0.600_{-0.020}^{+0.020}$ & $0.144_{-0.011}^{+0.011}$ \\
\hline Local & $-9.9_{-1.2}^{+1.2}$ & $-0.111_{-0.048}^{+0.048}$ & $0.239_{-0.033}^{+0.033}$ & $-7.0_{-0.6}^{+0.6}$ & $0.002_{-0.023}^{+0.023}$ & $0.174_{-0.016}^{+0.016}$ \\
\hline Perseus & $-6.2_{-1.6}^{+1.6}$ & $-1.74_{-0.09}^{+0.09}$ & $0.490_{-0.065}^{+0.066}$ & $-7.0_{-1.2}^{+1.2}$ & $-1.54_{-0.05}^{+0.05}$ & $0.630_{-0.035}^{+0.036}$ \\
\hline Outer-1 & $-5.2_{-2.8}^{+2.8}$ & $-3.94_{-0.17}^{+0.17}$ & $0.437_{-0.147}^{+0.146}$ & $-9.7_{-1.1}^{+1.1}$ & $-3.85_{-0.07}^{+0.07}$ & $0.368_{-0.055}^{+0.055}$ \\
\hline Outer-1a & & & & $-8.1_{-1.7}^{+1.7}$ & $-5.81_{-0.13}^{+0.13}$ & $(0.00)$ \\
\hline Outer-2 & $-8.3_{-2.6}^{+2.6}$ & $-7.54_{-0.55}^{+0.54}$ & $(0.00)$ & $-9.2^{+2.0}+2.0$ & $-7.70_{-0.27}^{+0.27}$ & $(0.00)$ \\
\hline
\end{tabular}

Table 2. Differences between the parameters of segments traced by old and young Cepheids. $N_{\mathrm{o}}$ and $N_{\mathrm{y}}$ denote the numbers of objects that trace the old and young segments, respectively. $\Delta i_{\mathrm{o}-\mathrm{y}}$ expresses the difference between the pitch angles and $\Delta X_{\mathrm{s}, \mathrm{o}-\mathrm{y}}$ signifies the difference between the $X_{\mathrm{s}}$ values of the old and young segments

\begin{tabular}{lcccc}
\hline Segment & $N_{\mathrm{o}}$ & $N_{\mathrm{y}}$ & $\Delta i_{\mathrm{o}-\mathrm{y}}$ & $\Delta X_{\mathrm{s}, \mathrm{o}-\mathrm{y}}(\mathrm{kpc})$ \\
\hline Scutum & 6 & 17 & $2.8 \pm 2.3$ & $-0.11 \pm 0.07$ \\
Sagittarius-1 & 44 & 27 & $4.8 \pm 1.6$ & $-0.05 \pm 0.06$ \\
Sagittarius-2 & 94 & 21 & $3.5 \pm 0.9$ & $-0.099 \pm 0.030$ \\
Local & 66 & 28 & $2.9 \pm 1.3$ & $+0.113 \pm 0.053$ \\
Perseus & 178 & 33 & $-0.8 \pm 2.0$ & $+0.20 \pm 0.10$ \\
Outer-1 & 89 & 10 & $-4.5 \pm 3.0$ & $+0.09 \pm 0.18$ \\
Outer-2 & 7 & 4 & $-0.9 \pm 3.3$ & $-0.16 \pm 0.60$ \\
\hline
\end{tabular}



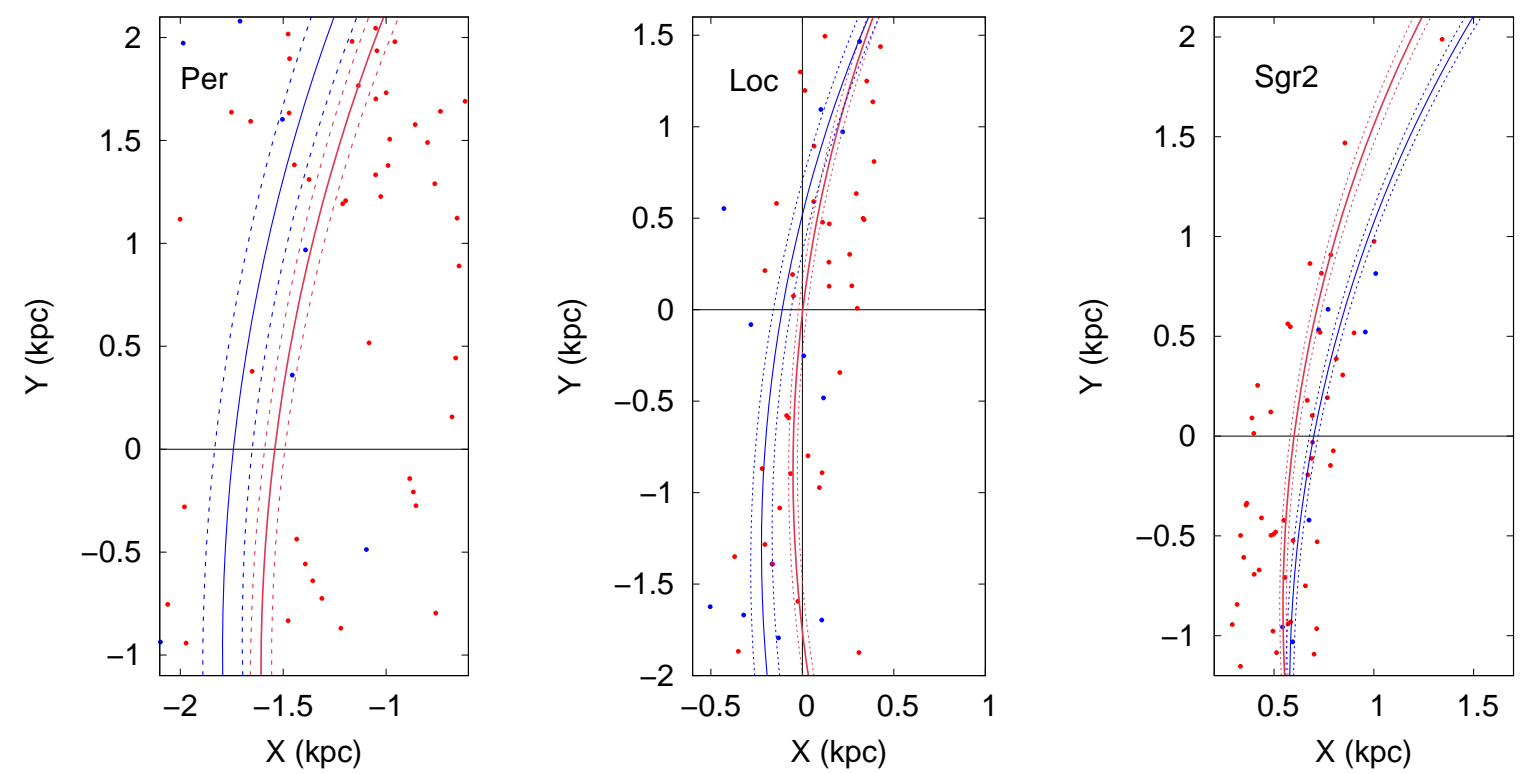

Fig. 4. $1 \sigma$ confidence regions for the Perseus, Local and Sagittarius-2 arms. Solid lines signify the center lines of segments, and dashed lines represent the boundaries of confidence regions. Old objects are displayed in red colour and young objects are shown in blue colour.

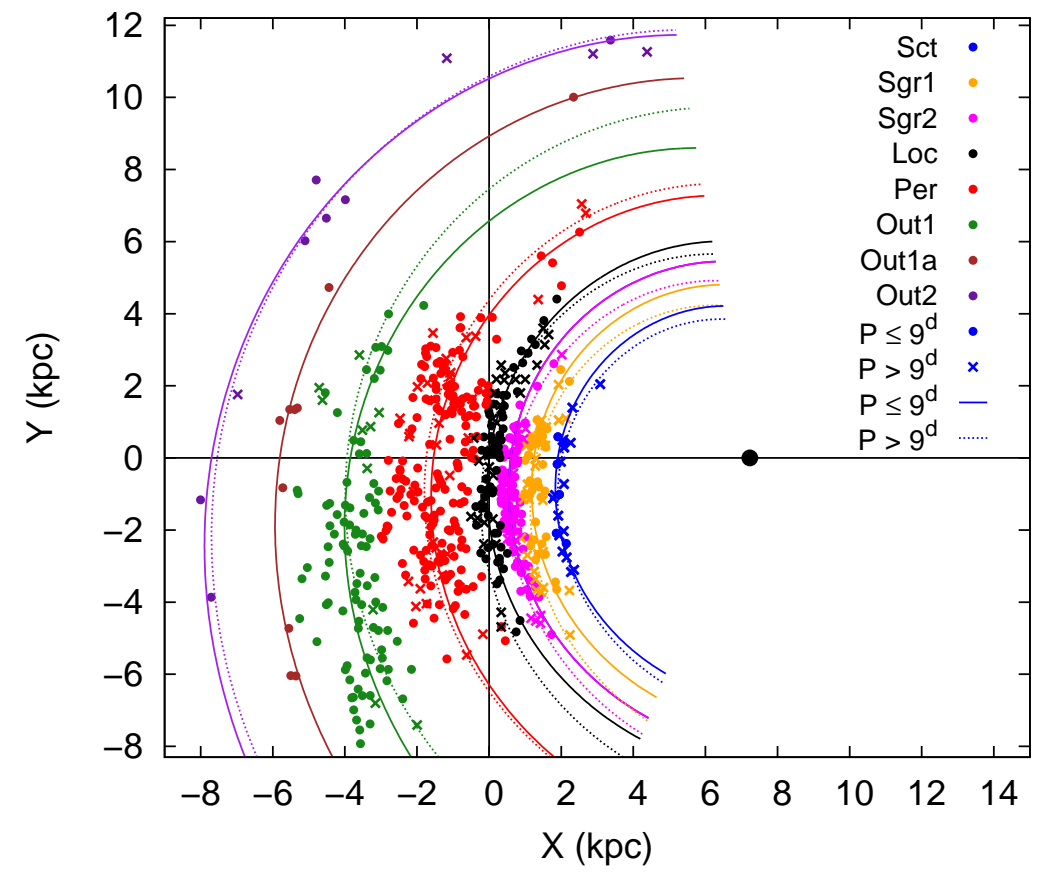

Fig. 5. Distribution of Cepheids and spiral segments projected on the Galactic plane $\left(R_{0}=\right.$ $7.23 \mathrm{kpc}$, see text). The young sample is represented by crosses, the old sample is marked by circles. Segments traced by young objects are represented by dotted lines, while those formed by the old sample are signified by solid lines. The Sun is placed at $X=0.0 \mathrm{kpc}, Y=0.0 \mathrm{kpc}$. The Galactic Center is located at $X=7.23 \mathrm{kpc}, Y=0.0 \mathrm{kpc}$. 


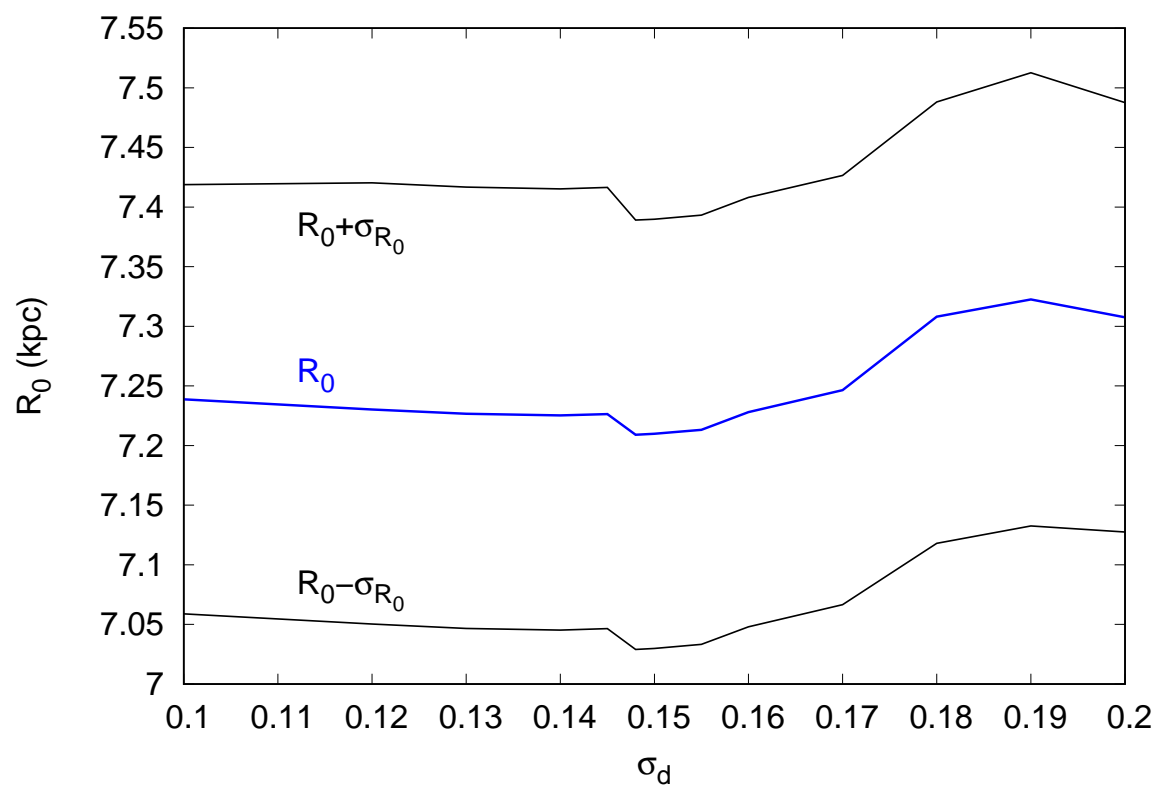

Fig. 6. Dependence of $R_{0}$ estimate on the assumed value of uncertainty of distance moduli. Blue line indicates the estimate obtained by optimizing parameters jointly for all segments, while black lines highlight the boundaries of the confidence interval of the $R_{0}$ estimate.

$\sigma_{d}$ of the distance moduli. One can see that the variation in the $R_{0}$ value does not exceed the statistical uncertainty of $R_{0}$ estimates. The $X_{\mathrm{s}}$ parameters and pitch angles for all segments also excibit no significant dependence on $\sigma_{d}$, therefore, we may conclude that the parameters of the center lines of segments are almost independent of the specific assumption about $\sigma_{d}$.

\section{Modeling the motion in a smooth Galactic potential}

In order to explain the bias between the spiral segments traced by young and old Cepheids, we modeled the motion of objects which were formed in a spiral arm segment. We chose the smooth potential of the Galaxy considered by Casetti-Dinescu et al. (2013). In that work, the disk was represented by the Miyamoto-Nagai model, the halo potential had a logarithmic dependence on a Galactocentric distance and the bar of the Galaxy was reproduced as an triaxial ellipsoid (the Ferrers potential) with a density profile varying by the power law. Casetti-Dinescu et al. (2013) implemented the Hernquist potential for reproducing the bulge component of the Galaxy. In Nikiforov \& Veselova (2020, in prep.) we have shown that this kind of model is not convenient for our investigation, so we considered three other kinds of bulge potential models. We chose the Plummer sphere (Kondrat'ev \& Orlov 2008), the Miyamoto-Nagai disk (Ninković 1992) and an isochrone potential. We tried to estimate the speed of a spiral pattern $\Omega_{\mathrm{p}}$ and the radial component $u$ of velocity given to a object by a spiral wave. For a square grid of the parameters in question, one calculates a $\chi$-squared function for the biases in longitude $\lambda_{\text {int }}$ of the intersection of the young segment and the old segment and in difference between the $X_{\mathrm{s}}$ 


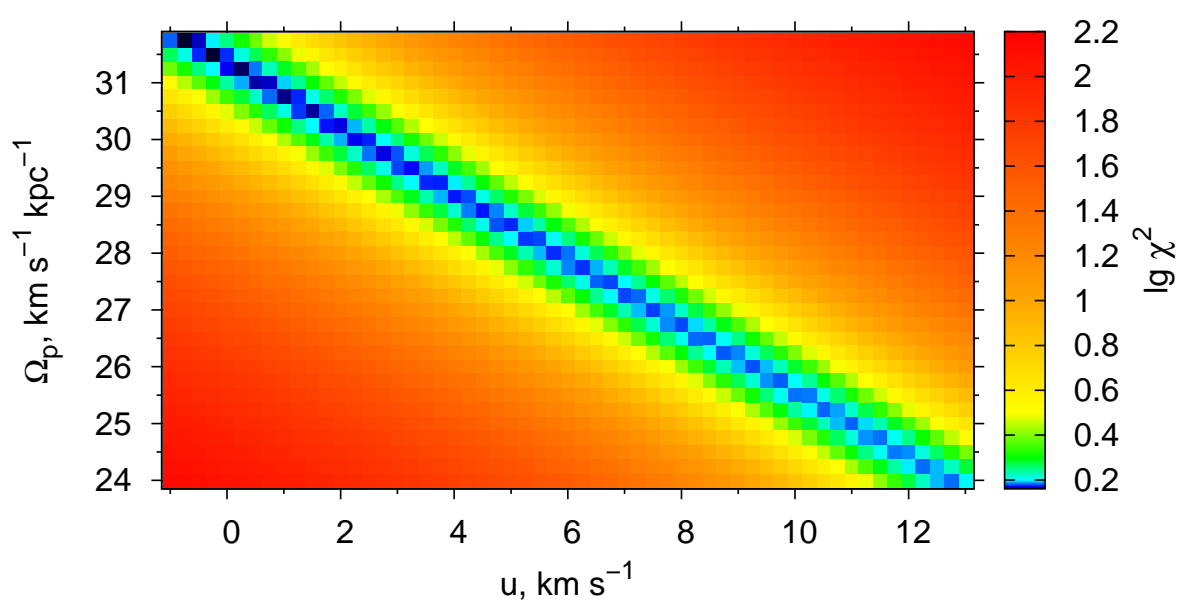

Fig. 7. Dependence of the decimal logarithm of the value $\chi^{2}$ on the values of the angular velocity of the spiral pattern $\Omega_{\mathrm{p}}$ and the component $u$ of the perturbation velocity of the spiral pattern for the Sagittarius-2 segment.

values for segments traced by young and old objects:

$$
\chi^{2}\left(u, \Omega_{\mathrm{p}}\right)=\frac{\left(\Delta X_{\mathrm{s}, \mathrm{obs}}-\Delta X_{\mathrm{s}, \mathrm{mod}}\right)^{2}}{\sigma^{2}\left(\Delta X_{\mathrm{s}}\right)}+\frac{\left(\lambda_{\text {int }, \mathrm{obs}}-\lambda_{\text {int }, \mathrm{mod}}\right)^{2}}{\sigma^{2}\left(\lambda_{\text {int }}\right)} .
$$

We ascertained that chosen kind of a bulge potential does not have a strong influence on results of modeling because the segments considered are not situated close to the central part of the Galaxy. We performed numerical simulations for two large segments. For both Sagittarius- 2 and Perseus segments $\Omega_{\mathrm{p}}$ and $u$ are strongly correlated and could not be estimated simultaneously. The dependence of $\chi^{2}$ on $\Omega_{\mathrm{p}}$ and $u$ for the Sagittarius-2 segment is depicted in Figure 7. One can see that if we take $u$ equal to 0 , we obtain too high value of $\Omega_{\mathrm{p}}\left(\sim 31 \mathrm{~km} \mathrm{~s}^{-1} \mathrm{kpc}^{-1}\right)$. If we take an estimate of $\Omega_{\mathrm{p}}=25.2 \pm 0.5 \mathrm{~km} \mathrm{~s}^{-1}$ obtained by Dambis et al. (2015), we get the value of $u$ equal to $10 \pm 1.5 \mathrm{~km} \mathrm{~s}^{-1}$.

\section{Variability of the pitch angles, influence on the pitch angles of the Sun-Galactic center distance, and the displacement of the pole of the spiral pattern}

Savchenko and Reshetnikov (2013) concluded that approximately $2 / 3$ of spiral galaxies manifest variations in the pitch angle of more than $20 \%$, so we investigated the question of variability in the pitch angle throughout the spiral segment. We considered the pitch angle linearly varying with the Galactocentric longitude $\lambda: i(\lambda)=i_{0}+i_{1} \cdot \lambda$. The only part of the likelihood function that is to be changed concerns the distance $w$ from a point to the center line of a segment. The equation for a longitude $\Lambda_{\bmod , j}$ of a point representing 

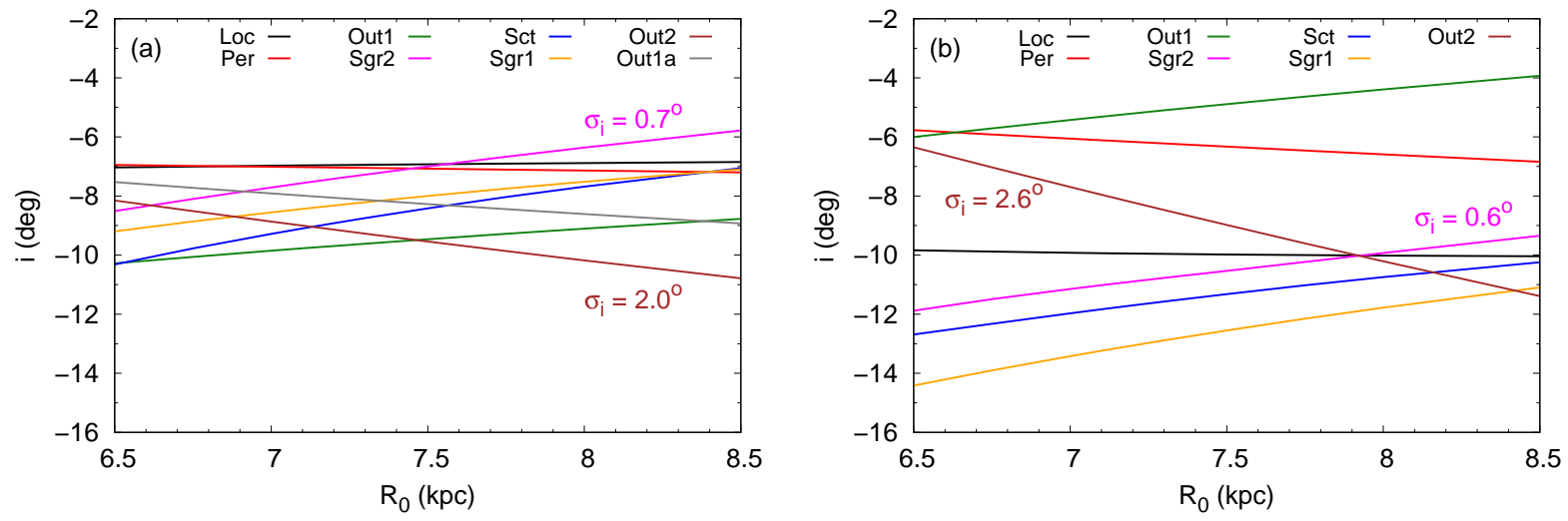

Fig. 8. Dependence of the pitch angles on $R_{0}$ for old (a) and young (b) segments. $\sigma_{i}$ denotes the characteristic value of the pitch angle uncertainty.

the base of a perpendicular drawn onto a center line of the $a$-th segment takes the form

$$
\begin{aligned}
& \left(X_{0, j}-R_{0}\right)\left(\sin \Lambda_{\mathrm{mod}, j}-\left[k_{a}+\frac{i_{1, a} \cdot \Lambda_{\mathrm{mod}, j}}{\cos ^{2} i_{a}}\right] \cos \Lambda_{\mathrm{mod}, j}\right)+Y_{0, j}\left(\cos \Lambda_{\mathrm{mod}, j}+\right. \\
& \left.+\left[k_{a}+\frac{i_{1, a} \cdot \Lambda_{\mathrm{mod}, j}}{\cos ^{2} i_{a}}\right] \sin \Lambda_{\mathrm{mod}, j}\right)-\left[k_{a}+\frac{i_{1, a} \cdot \Lambda_{\mathrm{mod}, j}}{\cos ^{2} i_{a}}\right]\left|R_{0}-X_{\mathrm{s}, a}\right| e^{k_{a} \cdot \Lambda_{\mathrm{mod}, j}}=0,
\end{aligned}
$$

where $k_{a}\left(\Lambda_{\bmod , j}\right) \equiv \tan \left(i_{a}\left(\Lambda_{\bmod , j}\right)\right)=\tan \left(i_{0, a}+i_{1, a} \cdot \Lambda_{\bmod , j}\right)$. Equation (12) is similar to (7), and all the difference is in the multiplier that occurs due to the variability in the pitch angle.

We obtained the spiral segment parameters for young and old subsystems simultaneously by minimizing the $\mathfrak{L}$ function (4) taking into account the new equation (12) for determining $w$. We found that no segment has a significant value of $i_{1}$. Therefore, this system of segments can be described by spiral segments with the constant pitch angles.

It was also interesting to investigate the dependence of the pitch angles on the assumed value of $R_{0}$. We fixed the $R_{0}$ values and optimized the other parameters of segments. Figure 8 shows the dependence of the pitch angle on $R_{0}$ for young and old segments. One can notice that although the spread of the pitch angle values for young segments is greater than that for old ones, the slope of the dependency on $R_{0}$ is generally the same for young and old segments. For several segments pitch angles change significantly over the considered range of $R_{0}$ values. For example, the pitch angle of both the young and old Sagittarius-2 segments changes by more than 2.5 , while $3 \sigma_{i}$ does not exceed $2^{\circ} .1$. On the contrary, the pitch angles of Outer- 2 segments do not vary significantly because of the large uncertainty. Figure 8 demonstrates that in general the value $R_{0}$ can significantly affect the estimates of the pitch angles.

We also tried to clarify if a bias exists in the pole of the spiral pattern from the axis on which the Galactic longitude is equal to 0 . We consider the coordinates of a pole to be $\left(X_{0}, Y_{0}\right)$ (see Fig. 9). Therefore, the solar Galactocentric distance should be calculated as $R_{0}=\sqrt{X_{0}^{2}+Y_{0}^{2}}$. We also can derive an angular bias of a pole: $\theta=\arctan \left(Y_{0} / X_{0}\right)$.

As in the case of variable pitch angle, only the equation for determining the distance 


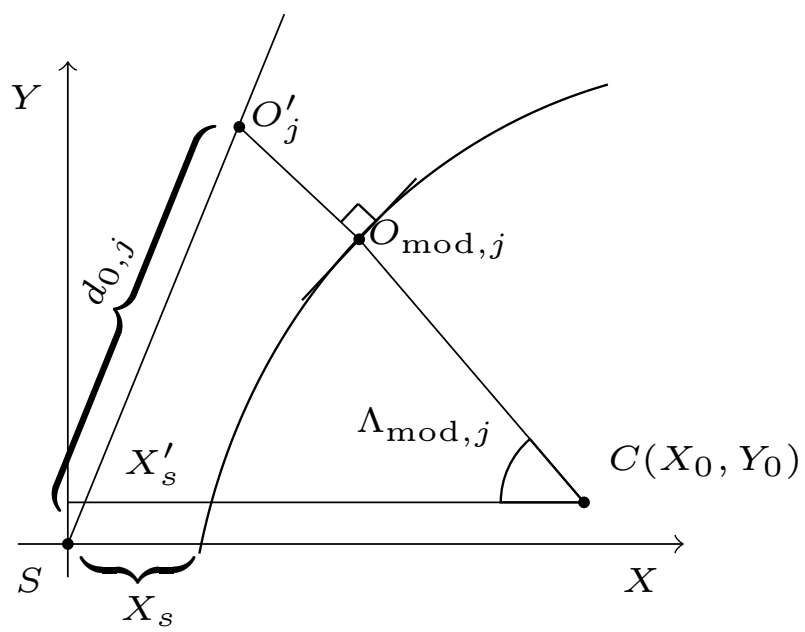

Fig. 9. Location of an object relative to the center line of the spiral segment with a pole offset from the line $S X$ of zero Galactic longitude (the nominal direction to the Galactic center). $C$ signifies the spiral pole, and $X_{\mathrm{s}}^{\prime}$ is the $X_{\mathrm{s}}$ value corrected for the shift of $C$. $O_{j}^{\prime}$ represents the reduced position for the $j$-th object, $O_{\bmod , j}$ is the point on a center line for which the distance $w$ from reduced position is minimal and $\Lambda_{\bmod , j}$ is the Galactocentric longitude of the point $O_{\bmod , j}$.

from the reduced position to the center line of the segments changes significantly and takes the form

$$
\begin{aligned}
\left(X_{0, j}-X_{0}\right)\left(\sin \Lambda_{\mathrm{mod}, j}-k \cos \Lambda_{\mathrm{mod}, j}\right) & -k\left|X_{0}-X_{\mathrm{s}}^{\prime}\right| e^{k \Lambda_{\mathrm{mod}, j}}+ \\
& +\left(Y_{0, j}-Y_{0}\right)\left(k \sin \Lambda_{\mathrm{mod}, j}+\cos \Lambda_{\mathrm{mod}, j}\right)=0
\end{aligned}
$$

where $X_{\mathrm{s}}^{\prime}$ represents the coordinate of the intersection point of the segment's central line with the line that is parallel to the $X$-axis and passes through the pole of the spiral pattern (see Fig. 9). Simultaneous optimization of parameters for young and old segments yielded the following estimate of the pole coordinates: $X_{0}=7.22 \pm 0.19 \mathrm{kpc}, Y_{0}=-0.06 \pm 0.15 \mathrm{kpc}$. Therefore, the bias of the pole is not significant and the angular bias $\theta=-0.5 \pm 1.3$ also can be treated as zeroth.

\section{Conclusions}

We divided the sample of Cepheids into relatively young and old subsystems and considered the spiral pattern consisting of 15 separate segments (seven traced by young objects and eight traced by old ones). Estimates of the parameters were obtained jointly for the two subsystems of segments following the maximum likelihood method for the spatial modeling of spiral arm segments which takes into account the natural dispersion across the segment and the uncertainty in the distance moduli. The obtained estimate $R_{0}=7.23_{-0.18}^{+0.19} \mathrm{kpc}$ in the modern LMC calibration (de Grijs et al. 2014) corresponds to the value $R_{0}=\left.\left.8.08_{-0.20}^{+0.21}\right|_{\text {stat. }}{ }_{-0.36}^{+0.38}\right|_{\text {cal }}$ kpc, which is consistent with modern estimates such as $R_{0}=8.34 \pm 0.18 \mathrm{kpc}$ obtained by Xu et al. (2018) from kinematics of $\mathrm{O}$ stars and masers, $R_{0}=8.178 \pm 0.013$ (stat.) \pm 0.022 (sys.) kpc (GRAVITY Collaboration et al. 2019) and $R_{0}=7.946 \pm 0.050$ (stat) \pm 0.032 (sys) kpc (Do et al. 2019) from modelling S0-2 orbit. 
For Perseus and Sagittarius-2 segments a significant difference between the pitch angles and $X_{\mathrm{s}}$ values was found, and confidence regions for this segments also show the difference between central lines. To interpret this displacement for Perseus and Sagittarius2 objects we carried out the dynamic modeling of the motion of objects which were formed in a spiral arm segment. At $\Omega_{\mathrm{p}}=25.2 \pm 0.5 \mathrm{~km} \mathrm{~s}^{-1} \mathrm{kpc}^{-1}$ (Dambis et al. 2015), the observed displacement can be explained by the value $u=10 \pm 1.5 \mathrm{~km} \mathrm{~s}^{-1}$.

We demonstrated that spiral segments traced by Cepheids do not possess a significant gradient in the pitch angle. Also the set of logarithmic segments do not need the pole of the spirals to be displaced, so the location of the geometric pole of the spiral pattern is consistent with the conventional direction to the Galactic center.

\section{ACKNOWLEDGMENTS}

A.V.V. acknowledges support from the Russian Science Foundation, Grant No. 1812-00050. We are grateful for the helpful comments from the anonymous referee.

\section{REFERENCES}

1. Berdnikov, L. N., Dambis, A. K., \& Vozyakova, O. V. 2000, AAS, 143, 211.

2. Bobylev, V.V., \& Bajkova, A.T. 2014, MNRAS, 437, 1549.

3. Braga, V. F., Bhardwaj, A., Contreras Ramos, R., Minniti, D., Bono, G., de Grijs, R., Minniti, J. H., \& Rejkuba, M. 2018, A\&A, 619, A51.

4. Casetti-Dinescu, D. I., Girard, T. M., Jílková, L., et al. 2013, AJ, 146, 33.

5. Do, T., Hees, A., Ghez, A., Martinez, G. D., Chu, D. S., et al. 2019, Science, 365, 664 .

6. Dambis, A. K., Berdnikov, L. N., Efremov, Yu. N., Kniazev, A. Yu., Rastorguev, A. S., Glushkova, E. V., Kravtsov, V. V., Turner, D. G., Majaess, D. J., \& Sefako, R. 2015, Astron. Lett., 41, 489.

7. de Grijs, R., Wicker, J. E., \& Bono G. 2014, AJ, 147, 122.

8. Efremov, Yu. N. 2003, ARep, 47, 1000.

9. Efremov, Yu. N. 2011, ARep, 55, 108.

10. Englmaier, P., Pohl, M., \& Bissantz, N. 2011, MSAIS, 18, 199.

11. Francis C., \& Anderson, E. 2012, MNRAS, 422, 1283.

12. GRAVITY Collaboration (Abuter R. et al.), 2019, Astron. Astrophys., 625, L10

13. Joshi, Y. C., \& Joshi, S. 2014, NewAst, 28, 27. 
14. Karimova, D. K., \& Pavlovskaya, E. D. 1974, Soviet Astronomy, 17, 470.

15. Kondrat'ev, A. S., \& Orlov, V. V. 2008, Astron. Lett., 34, 537.

16. Lépine, J. R. D., Mishurov, Yu. N., \& Dedikov, S. Yu. 2001, ApJ, 546, 234.

17. Mel'nik A. M., Rautiainen P., Berdnikov L. N., Dambis A. K., \& Rastorguev A.S. 2015, Astron. Nachr., 336, 70.

18. Nikiforov, I., \& Veselova, A. 2015, BaltA, 24, 387

19. Nikiforov, I. I., \& Veselova, A. V. 2018a, Astron. Lett., 44, 81.

20. Nikiforov, I. I., \& Veselova, A. V. 2018b, Astron. Lett., 44, 763.

21. Ninković, S. 1992, Astron. Nachr., 313, 83.

22. Savchenko, S. S., \& Reshetnikov, V. P. 2013, MNRAS, 436, 1074.

23. Vallée, J. P. 2008, AJ, 135, 1301.

24. Vallée, J. P. 2015, MNRAS, 450, 4277.

25. van de Hulst H.C., Muller C.A., \& Oort J.H. Bull. Astron. Inst. Netherl., 1954, 12, 117.

26. Veselova A. V., \& Nikiforov, I. I. 2018, in MSA Conf. Abstr. Vol. 1, eds. O. Yu. Malkov, A. S. Rastorguev, N. N. Samus' (Moscow: IZMIRAN), 100.

27. Xu, Y., Hou, L.-G., \& Wu, Y.-W. 2018, RAA, 18, 146. 